\title{
Neuroimaging of Ischemia and Infarction
}

\author{
Erica C. Sá de Camargo*广 and Walter J. Koroshetz* \\ *Massachusetts General Hospital, Boston, Massachusetts 02114; and ${ }^{\dagger}$ Hospital das Clínicas da Faculdade de Medicina da \\ Universidade de São Paulo, São Paulo 05403-000, Brazil
}

\begin{abstract}
Summary: Since the introduction of thrombolytic therapy as the foundation of acute stroke treatment, neuroimaging has rapidly advanced to empower therapeutic decision making. Diffusionweighted imaging is the most sensitive and accurate method for stroke detection, and, allied with perfusion-weighted imaging, provides information on the functional status of the ischemic brain. It can also help to identify a response to thrombolytic and neuroprotective therapies. Additionally, multimodal magnetic resonance imaging, including magnetic resonance angiography, offers information on stroke mechanism and pathophysiology that can guide
\end{abstract}

long-term medical management. Multimodal computed tomography is a comprehensive, cost-effective, and safe stroke imaging modality that can be easily implemented in the emergency ward and that offers fast and reliable information with respect to the arterial and functional status of the ischemic brain. Accessibility, contraindications, cost, speed, and individual patient-determined features influence which is the best imaging modality to guide acute stroke management. Key Words: Stroke, cerebral infarction, computed tomography, magnetic resonance imaging, positron emission tomography, spectroscopy.

\section{INTRODUCTION}

In the decade revolutionized by thrombolytic treatment of acute ischemic stroke, neuroimaging has become one of the most powerful tools in cerebrovascular disease therapy and can offer neurologists and emergency physicians critical information for proper management of acute stroke patients, such as: $:^{1,2}$

1. Accurate identification of ischemic stroke (exclusion of hemorrhage and stroke mimics).

2. Identification of arterial stenoses and occlusions.

3. Prediction of likely stroke severity, i.e., differentiating large infarcts in eloquent regions from smaller infarcts in "silent" brain regions.

4. Identification of patients with vascular lesions amenable to endovascular or surgical treatments.

5. Assessment of persistent abnormal perfusion and arterial occlusion versus reperfusion and arterial recanalization after therapy, and visualization of the collateral arterial blood flow.

6. Insight into the possible mechanisms of stroke, directing long-term preventive strategies.

Research is currently underway to enable clinicians to

Address correspondence and reprint requests to Walter J. Koroshetz, M.D., Massachusetts General Hospital, Department of NeurologyVBK 915, 55 Fruit Street Boston, MA 02114-2622. E-mail: wkoroshetz@partners.org. emergently identify tissue that has already infarcted from ischemic tissue still salvageable by reperfusion strategies. We will now outline the main imaging modalities presently available for stroke imaging and their individual strengths and potential pitfalls. Special attention will be given to x-ray computed tomography (CT) and magnetic resonance imaging (MRI), which are currently the most frequently used techniques in acute stroke assessment.

\section{NEUROIMAGING MODALITIES}

\section{Positron emission tomography}

The reader is referred to an excellent review on PET imaging that has been published separately in the current volume. ${ }^{3}$ Due to its ability to quantitatively measure important metabolic variables, PET is considered the gold-standard imaging method to assess definitive neuronal loss and potentially salvageable tissue (penumbra) in brain ischemia. With PET parameters, the ischemic penumbra has been shown to have reduced cerebral blood flow $(\mathrm{CBF})$, elevated oxygen extraction fraction, normal oxygen metabolic rate $\left(\mathrm{CMRO}_{2}\right)$, and preserved ${ }^{11} \mathrm{C}$-flumazenil (FMZ) binding. Thresholds for tissue viability have been determined with measurements of CBF and $\mathrm{CMRO}_{2}$, but a potential limitation to the use of these cojoined variables is the need for arterial blood sampling. 

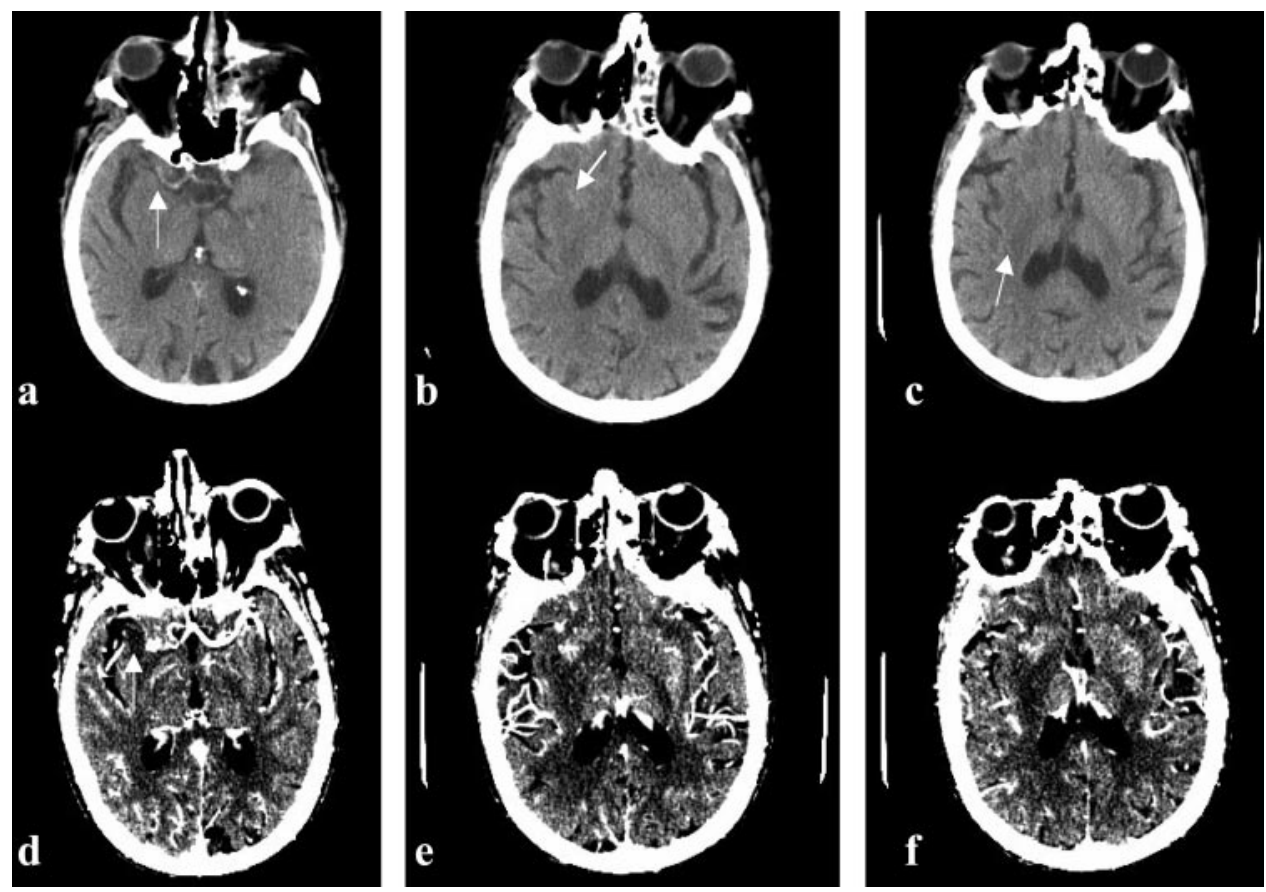

FIG. 1. Seventy-nine-year-old right-handed female, with $5 \mathrm{~h}$ onset of left-sided hemiplegia and neglect, right gaze preference, NIHSS $=15$. This figure demonstrates EIC. a) Dense vessel sign in projection of right M1 branch (arrow); b) Right insular ribbon sign (arrow). c) Hypoattenuation of right lentiform nucleus (arrow). Panels d-f are the corresponding CT angiography source images demonstrating increased conspicuity of the EIC seen in this patient. Note the filling defect of the right M1 branch in panel d (arrow).

${ }^{11} \mathrm{C}-\mathrm{FMZ}$ is a marker of cortical neuronal integrity and can reliably offer relative quantitative thresholds for cortical ischemic injury. ${ }^{4}$ Compared with diffusionweighted imaging (DWI), ${ }^{11} \mathrm{C}-\mathrm{FMZ}$ is equivalent in the prediction of cortical ischemic infarction but has a lower probability of false positives than DWI. ${ }^{5}$ Another tracer that has been used in PET stroke imaging is 18-F-fluoromisonidazole. It binds preferentially to hypoxic but viable tissue in the periphery of the infarct or in close peri-infarct regions. ${ }^{6}$ The main limitation to the use of PET in stroke assessment is its limited availability in most stroke centers, particularly in the emergency setting. The main advantage is its ability to map important metabolic consequences of abnormal perfusion, i.e., ischemia.

\section{X-ray computed tomography}

The advantages of CT scanning of brain ischemia and infarction are its speed, cost effectiveness, widespread availability, and ability to identify clinically significant hemorrhage. Recently, an expanded CT imaging protocol improved the ability to detect early ischemic changes (EIC). Noncontrast CT (NCCT), together with CT angiography (CTA), has been shown to be a safe, feasible, and fast method (on and off the table in less than $30 \mathrm{~min}$ ) to image the large vessels both intra- and extracranially and identify EIC. ${ }^{7}$ Safety has improved with the advent of nonionic contrast materials: in an experimental animal model, nonionic contrast agents did not affect infarction volume or neurological performance in the acute phase of middle cerebral artery (MCA) stroke; ${ }^{8}$ in a case control study, Palomaki et al. ${ }^{9}$ reported that the use of nonionic contrast agents in acute stroke did not affect clinical outcomes; Smith et al. ${ }^{7}$ have demonstrated the safety profile of a NCCT/CTA protocol for acute stroke patients.

\section{Noncontrast CT}

NCCT is usually the first neuroimaging examination performed in acute stroke assessment. In addition to detecting hemorrhage, modern NCCT can reveal EIC, such as: hypoattenuation of the parenchyma and gray matter with loss of gray-white differentiation (insular ribbon sign, obscuration of the lentiform nucleus, brain swelling with sulcal effacement) and compression of the ventricular system and basal cisterns, the dense artery sign and the MCA-dot sign (FIGS. 1, a-c, and 3a). ${ }^{10-13}$ EIC have been reported in 31-53\% of cases within $3 \mathrm{~h}$ of stroke onset. ${ }^{14,15}$ Physiologically, the NCCT hypodensities of acute infarction represent water uptake in the ischemic brain tissue as shown in a recent rat model study of MCA occlusions, in which a $1 \%$ increase in net brain water content corresponded to a reduction in x-ray attenuation of 1.8 Hounsfield units (HU). ${ }^{16}$ CT hypoattenuation has a significant correspondence to critically hypoperfused tissue as seen by PET. ${ }^{17}$ These early hypodensities have been shown to have a positive predictive value for stroke as seen on follow-up CT scans of $87 \% .{ }^{14}$ A hyperdense MCA (HMCA) sign and the more recently described MCA-dot sign are less sensitive for 

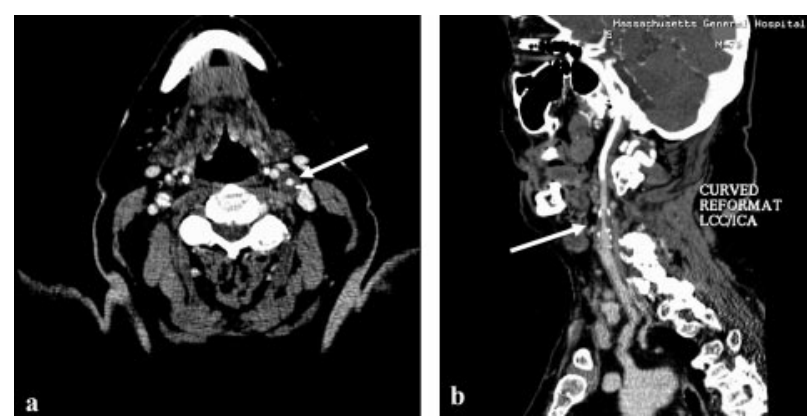

FIG. 2. Seventy-six-year-old male, presenting recurrent transient episodes of right-sided weakness and word-finding difficulties. CTA shows moderate to severe stenosis of the left internal carotid artery (arrows), which is better visualized on the axial source-images (a) than on the MIP images (b).

angiographic MCA occlusions (39\% and 38\% sensitivity, respectively) although highly specific (95\% and $100 \%$ specificity, respectively). ${ }^{18}$

Different approaches have been proposed for proper assessment of EIC, due to their implications for patient outcome and triage to thrombolysis. The $>1 / 3 \mathrm{MCA}$ territory rule is controversial but has been extensively used in clinical practice to exclude patients for thrombolytic treatment. ${ }^{19}$ In the ECASS I study, a more than one-third MCA territory hypodensity corresponded to a higher risk of fatal hemorrhage after thrombolysis and worse clinical outcomes, although the timeframe for thrombolysis was extended and a higher dose of recombinant tissue plasminogen activator (rt-PA) was used than in the National Institute of Neurological Disorders and Stroke (NINDS) trial. ${ }^{19,20}$ In the NINDS rt-PA trial, in which patients received thrombolysis within $3 \mathrm{~h}$ of stroke onset, patients with early CT signs of ischemia were still more likely to have good outcomes than patients who received placebo, even if the EIC were more than one-third of the MCA territory. ${ }^{14,20}$ Although the HMCA sign was associated with poor clinical outcome in ECASS I, these patients were more likely to have good neurological recovery if treated with rt-PA than placebo. ${ }^{19-21}$ In patients with MCA occlusions, the presence of EIC (within $5 \mathrm{~h}$ ) can have positive predictive values for fatal outcomes ranging from $32 \%$ for HMCA sign to $85 \%$ for hypodensity involving more than one-half the MCA territory. ${ }^{22}$ Early hypoattenuation of more than one-half of the MCA territory is an independent predictor of malignant infarction and neurologic deterioration. $^{23,24}$

The more recent Alberta Stroke Programme Early CT Score (ASPECTS) is a method of quantifying EIC in MCA strokes in 10 different regions in which, from a maximum total score of 10 , one point is deducted for each region presenting EIC. ${ }^{25}$ Baseline ASPECTS in MCA strokes within $3 \mathrm{~h}$ correlate inversely with the severity of the NIH stroke scale (NIHSS) and with functional outcome. ${ }^{25}$ Patients with a baseline ASPECTS greater than 7 that receive intra-arterial thrombolysis have significantly more independent functional outcomes at 90 days than patients with baseline ASPECTS of 7 or less. $^{26}$

EIC detection can be enhanced by using more narrow windows with soft-copy image interpretation, improving the sensitivity for EIC detection from 57\% with standard window settings (center level setting of $20 \mathrm{HU}$ and window-width of $80 \mathrm{HU}$ ) to $71 \%$ with high-contrast settings (center level setting of 28-36 HU and window-width of 1-30 HU), without loss of specificity. ${ }^{27}$

\section{Whole brain CT perfusion imaging}

An important advance in stroke imaging is the development of CT perfusion imaging (CTP) facilitated by the conversion to fast helical CT machines. After a bolus infusion of intravenous contrast, two data sets are obtained: 1) quantitative maps of mean transit time (MTT), $\mathrm{CBF}$, and cerebral blood volume (CBV), constructed from single 2-cm slabs of brain tissue per bolus of CT dye; and 2) raw images for CTA. ${ }^{28}$ The latter early contrast-enhanced images of whole brain we term "whole brain perfused blood volume" or "CTA-source images" (CTA-SI). In this technique, a 90-120 ml bolus of IV contrast material, infused at a rate of $2.5-3 \mathrm{ml} / \mathrm{sec}$, provides a steady state of the contrast during scanning. Images are obtained in two sequential acquisition groups: first, from the skull base to the vertex during maximum contrast enhancement, for whole brain perfusion imaging and the intracranial angiogram; second, from the aortic arch to the skull base, for imaging of the extracranial arteries. ${ }^{29}$

Contrast-enhanced images have better signal difference between normal and ischemic brain tissue than NCCT, showing increased conspicuity of the infarcted tissue (FIGS. 1, d-f, and 3b). ${ }^{28}$ Physiologically, CTA-SI represents cerebral blood volume that is reduced in the core infarct and correlates with infarct volume as seen on DWI and follow-up NCCT (FIG. 3, b and c). ${ }^{30-32}$ However, in a study comparing PET assessment of critical cortical hypoperfusion with CTA-SI, there was inaccuracy in the assessment of the volumes of critical perfusion deficits by means of CTA-SI. ${ }^{33}$

CTA-SI can help predict outcome after intra-arterial thrombolysis: successfully recanalized patients show a linear correlation between initial CTA-SI volumes and final infarct volume, whereas, for unsuccessful treatment, the final infarct volume is greater than on initial CTASI. ${ }^{30}$ Although exceptions occur, these data suggest that the lesion on CTA-SI is closely associated with the ischemic "core." In this way, the CTA-SI abnormality parallels the DWI abnormality on MRI. Both are signatures of significant ischemic injury but in some exceptional circumstances can be reversed by reperfusion. It has been shown that normalized perfusion cerebral blood volumes 

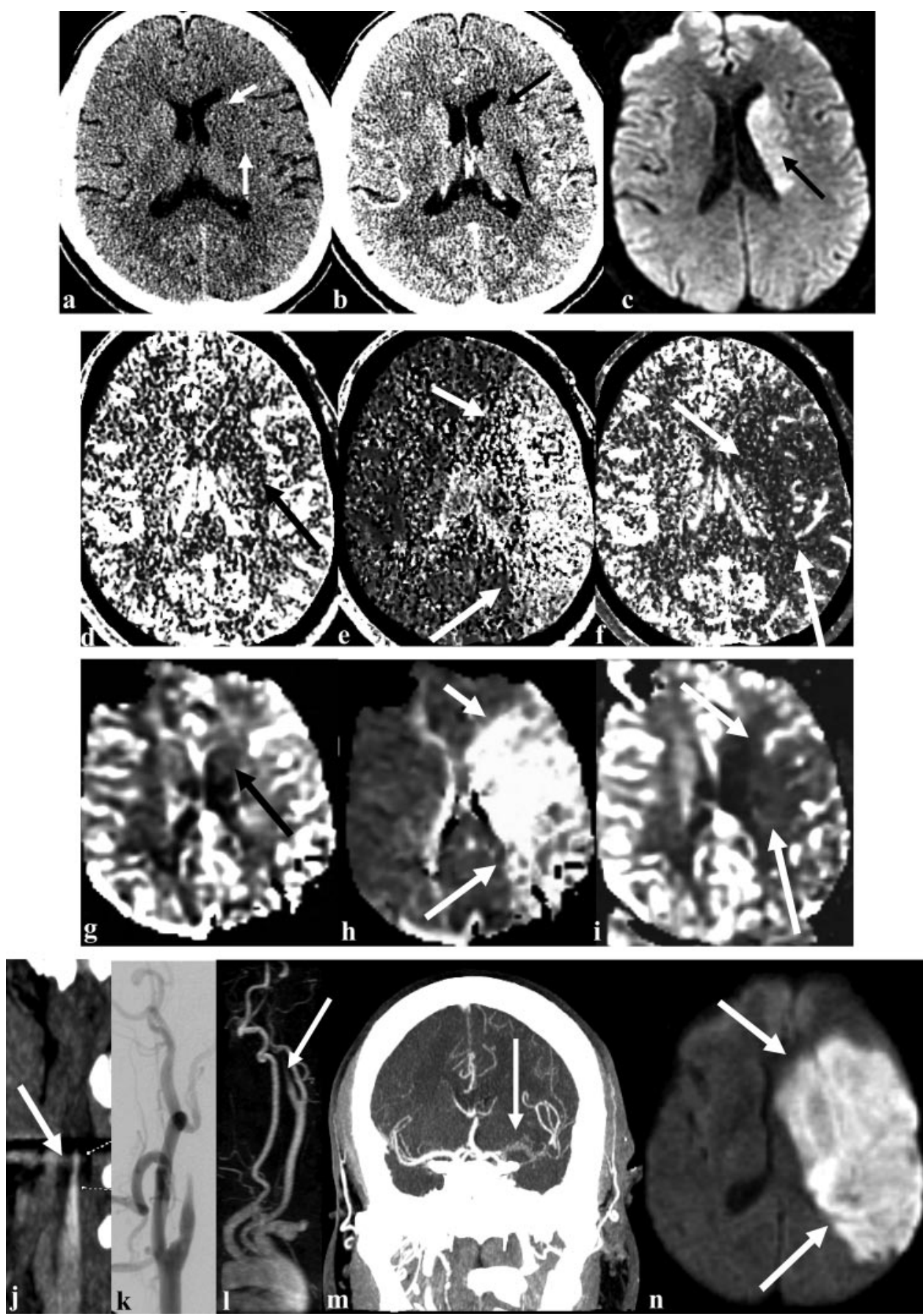

FIG. 3. Multimodality imaging in acute ischemic stroke. Top panel demonstrates obscuration of the caudate and putamen (arrows) in a patient with right hemiparesis and aphasia on noncontrast CT (a). CTA source image shows decreased vascular signal in the territory of the lenticulostriates (arrows) in the left hemisphere with increased vascular signal in the overlying cortex (b). DWI shows injury pattern (arrow) in the region that is abnormal on the CTA source images (c). Second panel demonstrates single slab CT perfusion study with low CBV (arrow) in the region abnormal on the DWI and CTA source image (d). MTT (e) and CBF (f) are abnormal throughout the left hemisphere congruous with the clinical deficit (arrows). Third panel demonstrates MR perfusion with CBV (g), MTT (h) and CBF (i) with findings identical to those demonstrated with CT perfusion (arrows). Bottom panel demonstrates the vascular lesions. Panel $\mathrm{j}$ is a CTA of the extracranial carotid showing beak like tapering characteristic of carotid dissection (arrow). Panel $k$ is a digital subtraction angiogram of the left carotid. Panel I is a MR angiogram demonstrating the same arterial obstruction in the left internal carotid artery (arrow). Panel $\mathrm{m}$ is a thick CT slice taken after a bolus of CT dye demonstrating the left middle cerebral artery clot on the coronal image (arrow). Note that there is no computer reconstruction, only a thick CT slice, which is available within $2-5$ min of imaging. Panel $n$ shows the final infarct that evolved to include the entire region that was abnormal on MR and CT bolus tracking perfusion scans (arrows). 


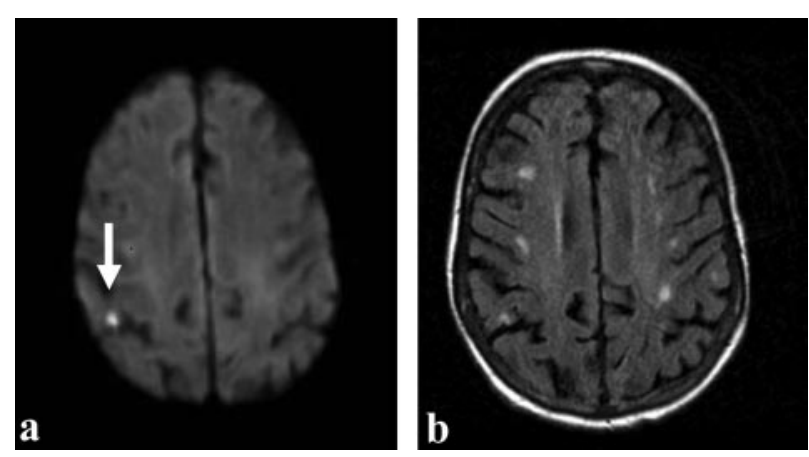

FIG. 4. Fifty-five-year-old right-handed female, with a previous history of atrial fibrillation, presented with sudden onset of leftsided weakness and dysarthria. MRI obtained $3 \mathrm{~h}$ after ictus shows: DWI bright lesion (a) in the right posterior parietal region, suggestive of an acute infarct (arrow). b: Corresponding axial FLAIR image demonstrates multiple subcortical white matter hyperintensities, suggestive of previous vascular insults.

at a threshold of $72 \%$ of the contralateral side have $11 \%$ probability of evolving to infarction, whereas at a threshold of $52 \%$, there is a $75 \%$ probability of infarction. A major advantage of CTA-SI is that computer processing is not necessary and the images are available immediately; in addition, the raw images for CTA are captured during the same scanning protocol.

\section{Quantitative brain CT perfusion imaging}

Innovative technology now makes it possible to obtain information about brain perfusion tissue through bolustracking contrast CT, such as CBF, CBV, and MTT. These three parameters are under study for their ability to distinguish infarcted tissue from potentially salvageable penumbra. Ischemic but noninfarcted tissue will have decreased CBF, elevated MTT, and normal or high CBV. Once infarcted, there will also be a persistent decrease in $\mathrm{CBV} .{ }^{34}$

Quantitative CTP can be obtained before or after CTA. Contrast must be infused at least at $4-10 \mathrm{ml} / \mathrm{s}$ through a large peripheral vein. CTP acquisition relies on the speed of helical scanners, which can image faster than one slice/sec and, therefore, can trace the contrast as it enters and washes out of a 2-cm slab of brain tissue. As opposed to MRI, there is a linear relationship between contrast concentration and signal intensity in HU. Contrast time-concentration curves are generated in an arterial region of interest, to provide the arterial input function (AIF). This is used to deconvolve the CBF in each pixel from the tissue time-concentration curve. $\mathrm{CBV}$ is the integral of the concentration-time curve, and MTT can be calculated from the central volume principle $(\mathrm{CBV}=\mathrm{CBF} \times \mathrm{MTT})$. The bolus tracking technique has difficulty differentiating effects due to delay or dispersion of the contrast bolus as compared to changes in the rate of blood flow.

The main limitation of this method is that its coverage is currently limited to one $2-\mathrm{cm}$ slab of brain per bolus.
With new generations of multidetector CT scanners, clinicians can anticipate CTP in thicker brain sections. Still, clinical knowledge of the most probable affected brain region should guide the selection of the brain slab to be scanned for CTP. Typically, the level of the basal ganglia is chosen because it is representative of the three main supratentorial arterial territories of the brain and because it contains an artery large enough to be used to calculate the AIF. ${ }^{29}$ Using the "toggling-table" technique, one can increase the anatomic coverage of CTP: during contrast bolus infusion, the scanner alternates between two distinct 2-cm regions, thus covering almost the full MCA territory, despite compromising temporal resolution. ${ }^{35}$ Another approach is to repeat scanning at a second level after infusion of a second contrast bolus. ${ }^{31}$ Recently, it has been shown that, by applying increased temporal scanning intervals with progressively higher doses of contrast agent, one can reduce the radiation dose without altering quantitative CTP assessment. ${ }^{36}$ CTP measurements of CBF have excellent correlation with xenon-CT$\mathrm{CBF}$ and single-photon emission CT (SPECT)-CBF maps in cerebrovascular diseases. ${ }^{37,38} \mathrm{CTP}$ maps have a sensitivity of $93 \%$ for hemispheric territorial infarcts but may miss brainstem and lacunar strokes. ${ }^{39}$ In acute stroke, using perfusion parameters with normalization to the unaffected contralateral hemisphere may show significant decreases in $\mathrm{CBV}$ and $\mathrm{CBF}$ and increases in MTT; in transient ischemic attack (TIA) patients, there may be reductions in CBF and increases in MTT, with normal CBV. ${ }^{40}$

CTP maps help predict outcomes in acute ischemic stroke. CBV below certain thresholds is a strong predictor of infarction despite recanalization. ${ }^{31} \mathrm{CBF}$ below certain thresholds is a strong predictor of infarction in the absence of recanalization. ${ }^{31}$ The mismatch between the $\mathrm{CBF}$ lesion and the small CBV lesion may be an indicator of tissue at risk. Within $12 \mathrm{~h}$ of stroke onset, CT-CBV abnormalities have a significant correlation with initial DWI and apparent diffusion coefficient (ADC) defects, as do normalized abnormal MTT and $\mathrm{CBF}$ values. ${ }^{41,42}$ However, CBV as measured with MRIperfusion-weighted imaging (PWI) maps have a more significant correlation with DWI volumes. ${ }^{41}$ Other authors have shown a direct correlation between all parameters of CT-CTP and PWI (Fig. 3, d-i). ${ }^{32}$

\section{CT angiography}

As described previously, with new helical and multidetector CT scanners it is possible to image the intracranial and extracranial arteries immediately after one bolus infusion of contrast and to obtain high-quality angiograms, which can be analyzed directly on raw axial source-images or on arterial reconstructions. Reconstructed images are generated using collapsed views of the imaging data, such as maximum intensity pixel (MIP) 
projections. The combined analysis of these two sets of images provides fast, noninvasive information about the arterial status in stroke patients. ${ }^{43}$ Compared to goldstandard digital subtraction angiography (DSA), CTA is highly specific (98\%), sensitive (98.4\%), and accurate (99\%) for the detection of intracranial stenoses or thrombi in stroke patients, with adequate inter-rater reliability. ${ }^{43,44}$ Intracranial CTA false positives have been reported, but it is debated whether these could be due to interval autolytic or mechanical lysis of thrombi during DSA ${ }^{43}$ Visualization of distal occlusions is limited with this method and is one of its major pitfalls. As for the assessment of carotid artery disease, a recent metanalysis comparing CTA with DSA showed CTA sensitivity and specificity of $85 \%$ and $93 \%$, respectively, for high-grade carotid stenosis, and $97 \%$ and $99 \%$, respectively, for carotid occlusion. ${ }^{45}$ Three-dimensional (3D) arterial reconstructions contribute to the detection of carotid disease, but raw CTA-SI are more accurate for the assessment of any degree of stenosis (FIG. 2). ${ }^{46}$

In patients who have contraindications to the use of iodinated dyes, gadolinium-enhanced CTA can alternatively be obtained. ${ }^{47}$ Bone artifacts can impede proper assessment of raw CTA-SI, especially when reviewing disease of the vertebral arteries. ${ }^{48}$ Nonetheless, CTA is now recognized as a first-choice, noninvasive tool in patient selection for thrombolysis.

\section{Multimodal CT protocol}

With the combined use of all the above mentioned CT modalities, one can obtain almost all the critical information needed for acute stroke therapy. The stepwise protocol that is used at our institution is:

1) NCCT: this first CT exam will help differentiate hemorrhagic from probable ischemic stroke and can reveal EIC which may or not contra-indicate acute thrombolysis.

2) CTA-SI and CTA: the second CT exam to be acquired; the raw CTA-source images can increase the conspicuity of the ischemic or already infarcted brain tissue. Just by reviewing the raw CTA-SI thick slices, one can search for large vessel arterial occlusions before the reconstructed CTA images are processed (FIG. 3m). Immediately after the CTA-SI is done, CTA arterial reconstructions (MIPS) can be obtained within 15-20 min and help to better localize intra- or extracranial arterial obstructions or occlusions.

3) CTP: immediately after CTA scanning and after a quick review of the thick CTA-SI images for arterial occlusions, the contrast-bolus CTP is obtained. The slice is chosen according to the presumed arterial territory involved. The decision is based both on the previous neuroimaging results and the clinical presentation. Thus, the chance of scanning at the correct level of a cerebral perfusion deficit is increased. Information about $\mathrm{CBV} /$
CBF or CBV/MTT mismatches will also be important in therapeutic decision making. Trials are in progress to test whether intravenous thrombolytic agents provide benefit to patients with "mismatch," even as long as $9 \mathrm{~h}$ after stroke onset.

This entire neuroimaging protocol can take up to 10-15 min to be obtained and an additional $20 \mathrm{~min}$ are required for the processing of the MIPS and CTP maps.

\section{Xenon-enhanced CT}

A variant $C T$ technique can be used to assess cerebral perfusion using xenon as a contrast agent. Xenon CT is obtained initially with the inhalation of stable xenon, an inert gas mixture of $28 \%$ xenon and $72 \%$ oxygen, which diffuses through the lung capillaries, reaches equilibrium in the blood and subsequently diffuses to bodily tissues. Dynamic CT brain scanning is performed during washout of xenon, and changes in brain attenuation during this process can be seen, producing quantitative and accurate CBF maps. ${ }^{49}$ This method requires good patient cooperation to perform xenon inhalation. In addition, rare side effects can occur, such as decreased respiratory rate, headache, nausea, vomiting and seizures.

\section{Magnetic resonance imaging}

Diffusion-weighted imaging. MRI has clearly been the most extraordinary new marker for stroke imaging in the past decade, mainly due to DWI and PWI. The technological foundation of DWI, PWI, and other advance MRI techniques has been reviewed separately in the current volume. ${ }^{42}$ In ischemic tissue, there is movement of water into cells that results in an overall reduced Brownian motion of water molecules. This is seen early on DWI images as hazy, hyperintense signals. DWI combines the T2-bright signal intensity seen in cytotoxic edema with additional hyperintensity due to decreased water diffusibility. Therefore, it is necessary to analyze maps of ADC to distinguish the effects of reduced water diffusibility (dark on ADC) from T2 "shine-through" (bright on ADC). Both features lead to the DWI bright signals seen in ischemia. As a result, infarcts on DWI have a very high signal to noise ratio. Because of a progressive increase in their $\mathrm{T} 2$ component, they tend to become progressively more intense within the first few days of stroke. After 1-2 weeks, ADC normalizes and then increases in the infarct; hence diffusion imaging not only serves as a sensitive detector of infarction but also tracks it temporally as a recent event (FIG. 4).

Sensitivity and specificity of DWI for stroke detection is very high. In a study of serial acute stroke patients, 411 underwent DWI imaging a mean $1.3 \pm 2.2$ days after stroke onset, and DWI showed $94 \%$ sensitivity and $96.6 \%$ specificity for stroke detection. In the subset of patients who underwent DWI within $6 \mathrm{~h}$ of stroke (122 patients), DWI was $97.3 \%$ sensitive and $100 \%$ specific and had much higher accuracy than CT and conventional 
MRI. However, after $12 \mathrm{~h}$, accuracy of DWI imaging was comparable with that of CT. There were 21 falsenegative DWI examinations in this study of which, after review, eight might have represented very early ischemia. ${ }^{50}$ In another study of 22 patients who underwent MRI and CT within $6 \mathrm{~h}$ of stroke-like symptoms (eight were stroke mimics), sensitivities and specificities for stroke detection were, respectively, $100 \%$ and $86 \%$ for DWI, $18 \%$ and $100 \%$ for conventional MRI, and $45 \%$ and $100 \%$ for $\mathrm{CT}^{51}$

The occurrence of false-negative DWI in stroke was reviewed in a cohort of 27 patients with acute stroke-like symptoms and negative initial DWI, of which 17 (63\%) had a final discharge diagnosis of stroke/TIA and six had follow-up imaging confirmation of infarction. Among these six strokes, three were lacunar and three were large-artery territory cortical infarctions, which at stroke onset presented abnormal MTT and CBF maps with normal CBV. ${ }^{52}$

DWI can also help identify the mechanism of stroke in some cases. In a study of 62 acute stroke patients presenting with classic lacunar syndromes, Ay et al. ${ }^{53}$ found that $10(16 \%)$ had multiple infarcts on DWI, and in four cases these involved multiple arterial territories; patients with multiple infarcts more frequently harbored an underlying embolic source than did patients with a single ischemic lesion. In a prospective study of 19 patients with a classic lacunar syndrome, alternate diagnoses (cortical, subcortical and restricted striato-capsular infarcts) were confirmed in 13 cases by the finding of multiple small embolic lesions. ${ }^{54}$ A larger study evaluated acute multiple brain infarcts as seen on DWI within 4 days of symptoms onset with relation to stroke mechanism in an Asian population; large-artery occlusion was significantly associated with multiple lesions in one or both sides of the anterior circulation or in the posterior circulation; cardioembolism was significantly associated with lesions in both anterior and posterior circulations; and small vessel occlusions were significantly and exclusively related to multiple lesions in both sides of the anterior circulation. ${ }^{55}$ Similar results have been replicated in a North American population. ${ }^{56}$

DWI bright signals do not necessarily represent irreversibly infarcted tissue but reflect redistribution of water from the extracellular to the intracellular space in ischemic tissue. Thresholds for infarction are also dependent upon duration and degree of ischemia, occurring at higher CBF levels with progressively prolonged ischemia. ${ }^{57}$ DWI reversibility immediately after early arterial reperfusion has been demonstrated in both animal models and in human MCA stroke, and correlates with clinical improvement in humans. ${ }^{58,59}$ However, in some cases, the DWI reversal is only temporary. ${ }^{59}$ Without arterial reperfusion, the DWI lesion will progressively enlarge over the first few days. ${ }^{60,61}$ DWI normalization occurs more frequently in the white matter and basal ganglia and is less likely to occur if there is a more severe initial drop in ADC. ${ }^{62}$

Perfusion-weighted MR imaging. MR perfusion weighted imaging is an important technological advancement in stroke imaging which, combined with DWI, provides highly relevant homodynamic information used for therapeutic decision-making in acute stroke. ${ }^{42} \mathrm{Com}$ bined DWI/PWI can also be a means of monitoring novel neuroprotective approaches.

Similar to CTP described above, bolus-tracking PWI is obtained using high-injection rate $(5 \mathrm{cc} / \mathrm{sec})$ echo planar imaging which can measure the passage of an intravenous bolus of paramagnetic contrast, such as gadolinium, through the capillary bed. ${ }^{63}$ Due to the high concentration of the contrast agent, T2 and susceptibility effects cause signal changes that can be visualized. To obtain adequate PWI maps, the AIF should ideally be obtained from the artery supplying the tissue at risk. This can be technically challenging, and the use of an inadequate AIF can increase the MTT, thus overestimating the tissue at risk of infarction. ${ }^{64}$ Echoplanar MR imaging is so fast that PWI can assess the hemodynamic status of the entire brain. To obtain more prompt semiquantitative measures, researchers compare the ischemic tissue with the contralateral unaffected hemisphere. PWI parameters have sensitivities for stroke assessment of 74-84\% (higher for CBF and MTT) and specificities of $96 \%$ for CBF and MTT, and $100 \%$ for CBV. ${ }^{65}$

The volumetric mismatch between the PWI and DWI volumes is a marker of potentially salvageable tissue at risk. ${ }^{66}$ Natural history studies show that in patients with mismatch, the DWI lesion will usually expand into the larger PWI lesion if reperfusion does not occur. ${ }^{60,67,68}$ This model has prompted the pursuit of therapies to prevent expansion of DWI lesions in patients with a clear DWI/PWI mismatch, even after the 3-h window for thrombolysis. DWI/PWI mismatch has been reported in up to $86.3 \%$ of acute stroke patients. ${ }^{69}$ Patients with mismatch have been selected for trials of reperfusion therapy after $3 \mathrm{~h} .{ }^{69 a}$ In patients with small subcortical lesions, the mismatch model does not seem to be of value because initial CBF lesions have been shown to be smaller than the corresponding ADC lesions. ${ }^{70}$ In patients with known large artery occlusion, a large "core" is such a strong predictor of poor outcome that the presence of mismatch may not be clinically relevant. ${ }^{71}$

Much debate revolves around which PWI parameter can better assess the severity and extent of ischemia and thus predict response to thrombolysis in acute stroke. Initial CBV volumes correlate more closely with the final stroke volume and with initial DWI. ${ }^{65,72}$ Therefore, mismatch is classically calculated comparing DWI with MTT or CBF maps. MTT and CBF commonly have a smaller correlation with final infarct volume than DWI 
and $\mathrm{CBV}$, and usually overestimate the final infarct volume (FIG. 3, g-i). ${ }^{65,68,72}$

Although sensitive to perfusion abnormalities, PWI lacks specificity. By normalizing PWI parameters relative to the opposite hemisphere, researchers have engaged in defining thresholds to define core, infarcted tissue, penumbra that infarcts, and penumbra that does not infarct. $\mathrm{CBF}$ appears to be the best parameter in this respect. It has been shown that normalized $\mathrm{CBF}$ in the core ranges from $0.26-0.32$; for penumbra that infarcts ranges are $0.42-0.46$; and for penumbra that remains viable, $0.58-0.62 .^{73,74}$ Grandin et al. ${ }^{75}$ found that within $6 \mathrm{~h}$ of stroke, the combined thresholds of CBF $35 \mathrm{ml} /$ $\min / 100 \times g$ and $\mathrm{CBV}$ of $8.2 \%$ were markers of evolution to infarction, with $81 \%$ sensitivity and $76 \%$ specificity, and that normalized MTT was always prolonged but not specific for prediction of tissue outcome. Threshold variability can be due to degree, duration, and location of ischemia, besides methodological inconsistencies. Conversely, other authors advocate the use of MTT as a marker for tissue viability. ${ }^{76-78}$ It has been shown that a time to peak or MTT more than 4-6 sec has a significant correlation with final infarct volume in acute stroke. ${ }^{77,78}$

The clinical application of this extraordinary technology aims at defying the present timeframe for thrombolytic therapy, and many studies have addressed this. Parsons et al. ${ }^{77}$ demonstrated that, in stroke patients with a DWI/PWI mismatch, final stroke volumes and clinical outcomes are significantly improved as compared with control stroke patients. This effect was most pronounced in mismatch tissue with MTT $>6 \mathrm{~s}$. Butcher et al. ${ }^{76}$ found that MTT was the most sensitive parameter for the prediction of tissue outcome in nonreperfused infarcts and that severe decreases in MTT were the best measure of response to reperfusion. Current clinical trials are underway investigating whether patients with mismatch benefit from intravenous thrombolytic therapy after $3 \mathrm{~h}$ from stroke onset. ${ }^{69}$

The use of PWI parameters has been advocated to evaluate response to therapy. Chalela et al. ${ }^{79}$ found that a reduction of $30 \%$ of initial MTT volumes $2 \mathrm{~h}$ after intravenous rt-PA was an early marker of long-term benefit of thrombolytic therapy. PWI can be predictive of outcomes even in the subacute phase of stroke, such as in monitoring response to induced hypertension with observed changes in the volumes of MTT maps. ${ }^{80}$

Susceptibility-weighted MRI (SWI). Susceptibilityweighted MRI utilizes magnetic artifacts generated by inhomogeneities of the magnetic field. Deoxyhemoglobin produces a nonuniform magnetic field, which accounts for signal changes seen in acute hemorrhages and for the blood oxygen level-dependent effect. With SWI, intraparenchymal hemorrhages can be seen within the first hour of bleeding, with high sensitivity and accuracy. ${ }^{81,82}$ SWI enables the visualization of multiple cere- bral microbleeds, which have been shown to be a risk factor for intracranial hemorrhage after stroke, both with and without thrombolytic therapy. ${ }^{83-85}$ How the presence of microbleeds seen on SWI should affect decisions to use thrombolytic agents has not been settled. Currently, SWI microbleeds should not be considered a contraindication to thrombolytic therapy in acute stroke. SWI is more reliable than CT in diagnosing intracranial hemorrhage after intra-arterial thrombolysis when CT hyperdensities may be due to dye extravasation and/or blood. ${ }^{86}$ Such information is highly significant in deciding when to institute antithrombotic therapy after endovascular procedures. Despite its high sensitivity for cerebral hemorrhage, studies are lacking that correlate SWI lesions, not seen on T2 MRI or CT scans, with clinically significant hemorrhage. SWI has other emerging applications for stroke imaging that surpass the scope of this manuscript. The interested reader is referred to the excellent review by Hermier and Nighoghossian. ${ }^{82}$

Magnetic resonance angiography. As with CT, MRI can provide high-quality assessment of intra- and extracranial macrovascular status in stroke. Time-of-flight (TOF) Magnetic resonance angiography (MRA), a gradient-echo technique, makes it possible to visualize the arterial lumen. 3D-TOF is preferred over two-dimensional (2D)-TOF because of its better signal-to-noise ratio and lower sensitivity for flow-voids. Axial images are reconstructed rendering maximum intensity projections of the arteries (FIG. 31). Images can be additionally empowered by a true flow effect with the use of gadolinium. Compared with DSA, 2D- and 3D-TOF MRA appear adequate for the diagnosis of carotid artery stenosis: sensitivity and specificity are $95 \%$ and $90 \%$, respectively, for high grade stenosis, and $98 \%$ and $100 \%$ for complete carotid occlusion. ${ }^{87}$ Some authors have shown, however, that 3D TOF flow-void detection thresholds vary according to the echo time chosen for the TOF pulse sequence, and the imaging hardware and software used could also interfere with these results. ${ }^{88}$ An important limitation of MRA is its limited capacity for the evaluation of leptomeningeal collaterals.

Accuracy for diagnosis of severe carotid stenosis seems to be similar for both gadolinium-enhanced MRA and 3D-TOF MRA. ${ }^{89}$ MRA has been shown to overestimate the degree of arterial stenosis as compared with other angiographic and ultrasound methods. However, for intracranial stenosis, there is a suggestion that gadolinium MRA is superior to conventional MRA in depicting low arterial flow and avoiding overestimation of vascular obstructions. ${ }^{90}$

\section{Magnetic resonance spectroscopy}

Magnetic resonance spectroscopy (MRS) is a noninvasive imaging method that can assess the concentration of biochemical markers of the brain in various disease 
states, including stroke. A comprehensive review of MRS, including technologic foundations and clinical applications is provided separately in the current volume. ${ }^{91}$ The more commonly measured chemicals are: 1) Lactate (Lac): produced in anaerobic metabolism, therefore a strong marker of cerebral ischemia, which may be persistently elevated in the chronic phase of stroke; 2) Nacetylaspartate: a marker of neuronal integrity, decreases in concentration in the acute stroke phase and may be persistently decreased in the subacute period; 3 ) Choline (Cho): present in lipids and increased in gliosis, and may both increase and decrease in acute stroke. Preliminary MRS data suggest that there may be pronounced metabolic variability within very low ADC regions in acute stroke. This may be a biochemical link to the reversible DWI phenomenon. ${ }^{92,93}$ Tissue viability may also be identified through Lac concentrations in the penumbral region. ${ }^{92}$ MRS can also help predict stroke outcome: acute stroke Lac/Cho ratios within the infarct core have been shown to have better correlations with final infarct volumes and clinical outcomes than DWI volumes. ${ }^{94}$

Diffusion tensor imaging. Diffusion tensor images (DTI) are composed of vectors that indicate the multiple directions of white-matter tracts. ${ }^{42}$ With the constraint of water diffusibility due to ischemia, water will preferentially diffuse along axons in the white matter. The direction of water diffusion can be estimated on a voxel-byvoxel basis, generating vector maps of white matter tractography. Despite its limited applicability in the evaluation of hyper-acute stroke, DTI can be useful in the evaluation of the effects of Wallerian degeneration, precise localization of subcortical strokes, prediction of stroke recovery, and planning rehabilitation in subcortical ischemia. ${ }^{95-97}$

\section{CONCLUSIONS}

Clearly, with the multiple imaging modalities available to date, it is possible to attend to the main goals of stroke imaging, as mentioned in the Introduction. Strokes can be well identified with multimodal CT, but DWI remains the most powerful and accurate method for stroke identification. Arterial occlusions are seen with CTA as well as with MRA. Both CTA and MRA are highly reliable, noninvasive methods to verify the results of thrombolytic therapy. CTP and DWI/PWI can rapidly provide functional information about brain perfusion and thus guide antithrombotic and neuroprotective strategies. Quantitative CTP is limited to a single slab of tissue per bolus, whereas MRI can provide whole brain $\mathrm{CBF}, \mathrm{CBV}$, and MTT. The goal of perfusion imaging is to reliably identify and distinguish brain tissue that is ischemic and will develop infarction without a specific intervention from brain tissue that is already damaged and cannot escape infarction.
Stroke imaging is still in its infancy, but advancements in this area are certain to guide therapeutic decision making in the near future. Studies are desperately needed to demonstrate whether advanced neuroimaging improves patient outcome in stroke.

Acknowledgments: Dr. Koroshetz is supported by HHS Grant AHRQ RO1 HS11392. Dr. Camargo is supported by a scholarship from Coordenação de Aperfeiçoamento de Pessoal de Nível Superior (CAPES)-Brazil.

\section{REFERENCES}

1. NINDS Group. Tissue plasminogen activator for acute ischemic stroke. N Engl J Med 333:1581-1587, 1995.

2. Furlan A, Higashida R, Wechsler L, Gent M, Rowley H, Kase C, et al. Intra-arterial prourokinase for acute ischemic stroke. JAMA 282:2003-2011, 1999.

3. Brooks DJ. Positron emission tomography and single-photon emission computed tomography in central nervous system development. NeuroRx 2:226-236, 2005.

4. Heiss WD, Kracht LW, Thiel A, Grond M, Pawlik G. Penumbral probability thresholds of cortical flumazenil binding and blood flow predicting tissue outcome in patients with cerebral ischaemia. Brain 124:20-29, 2001.

5. Heiss WD, Sobesky J, Smekal U, Kracht LW, Lehnhardt FG, Thiel A, et al. Probability of cortical infarction predicted by flumazenil binding and diffusion-weighted imaging signal intensity: a comparative positron emission tomography/magnetic resonance imaging study in early ischemic stroke. Stroke 35:1892-1898, 2004.

6. Markus R, Reutens DC, Kazui S, Read S, Wright P, Pearce DC, et al. Hypoxic tissue in ischaemic stroke: persistence and clinical consequences of spontaneous survival. Brain 127:1427-1436, 2004.

7. Smith WS, Roberts HC, Chuang NA, Ong KC, Lee TJ, Johnston $\mathrm{SC}$, et al. Safety and feasibility of a CT protocol for acute stroke: combined CT, CT angiography, and CT perfusion imaging in 53 consecutive patients. AJNR Am J Neuroradiol 24:688-690, 2003.

8. Doerfler A, Engelhorn T, von Kummer R, Weber J, Knauth M, Heiland $\mathrm{S}$, et al. Are iodinated contrast agents detrimental in acute cerebral ischemia? An experimental study in rats. Radiology 206: 211-217, 1998.

9. Palomaki H, Muuronen A, Raininko R, Piilonen A, Kaste M. Administration of nonionic iodinated contrast medium does not influence the outcome of patients with ischemic brain infarction. Cerebrovasc Dis 15:45-50, 2003.

10. Tomura N, Uemura K, Inugami A, Fujita H, Higano S, Shishido F. Early CT finding in cerebral infarction: obscuration of the lentiform nucleus. Radiology 168:463-467, 1988.

11. Truwit CL, Barkovich AJ, Gean-Marton A, Hibri N, Norman D. Loss of the insular ribbon: another early CT sign of acute middle cerebral artery infarction. Radiology 176:801-806, 1990.

12. Pressman BD, Tourje EJ, Thompson JR. An early CT sign of ischemic infarction: increased density in a cerebral artery. AJR Am J Roentgenol 149:583-586, 1987.

13. Barber PA, Demchuk AM, Hudon ME, Pexman JH, Hill MD, Buchan AM. Hyperdense sylvian fissure MCA "dot" sign: a CT marker of acute ischemia. Stroke 32:84-88, 2001.

14. Patel SC, Levine SR, Tilley BC, Grotta JC, Lu M, Frankel M, et al. Lack of clinical significance of early ischemic changes on computed tomography in acute stroke. JAMA 286:2830-2838, 2001.

15. Mohr JP, Biller J, Hilal SK, Yuh WT, Tatemichi TK, Hedges S, et al. Magnetic resonance versus computed tomographic imaging in acute stroke. Stroke 26:807-812, 1995.

16. Dzialowski I, Weber J, Doerfler A, Forsting M, von Kummer R. Brain tissue water uptake after middle cerebral artery occlusion assessed with CT. J Neuroimaging 14:42-48, 2004.

17. Grond M, von Kummer R, Sobesky J, Schmulling S, Rudolf J, Terstegge $\mathrm{K}$, et al. Early X-ray hypoattenuation of brain parenchyma indicates extended critical hypoperfusion in acute stroke. Stroke 31:133-139, 2000. 
18. Leary MC, Kidwell CS, Villablanca JP, Starkman S, Jahan R, Duckwiler GR, et al. Validation of computed tomographic middle cerebral artery "dot" sign: an angiographic correlation study. Stroke 34:2636-2640, 2003.

19. Hacke W, Kaste M, Fieschi C, Toni D, Lesaffre E, von Kummer R, et al. Intravenous thrombolysis with recombinant tissue plasminogen activator for acute hemispheric stroke. The European Cooperative Acute Stroke Study (ECASS). JAMA 274:1017-1025, 1995.

20. von Kummer R, Allen KL, Holle R, Bozzao L, Bastianello S, Manelfe C, et al. Acute stroke: usefulness of early CT findings before thrombolytic therapy. Radiology 205:327-333, 1997.

21. Manelfe C, Larrue V, von Kummer R, Bozzao L, Ringleb P, Bastianello S, et al. Association of hyperdense middle cerebral artery sign with clinical outcome in patients treated with tissue plasminogen activator. Stroke 30:769-772, 1999.

22. von Kummer R, Meyding-Lamade U, Forsting M, Rosin L, Rieke $\mathrm{K}$, Hacke W, et al. Sensitivity and prognostic value of early CT in occlusion of the middle cerebral artery trunk. AJNR Am J Neuroradiol 15:9-15, 1994.

23. Krieger DW, Demchuk AM, Kasner SE, Jauss M, Hantson L. Early clinical and radiological predictors of fatal brain swelling in ischemic stroke. Stroke 30:287-292, 1999.

24. Manno EM, Nichols DA, Fulgham JR, Wijdicks EF. Computed tomographic determinants of neurologic deterioration in patients with large middle cerebral artery infarctions. Mayo Clin Proc 78:156-160, 2003.

25. Barber PA, Demchuk AM, Zhang J, Buchan AM. Validity and reliability of a quantitative computed tomography score in predicting outcome of hyperacute stroke before thrombolytic therapy. ASPECTS Study Group. Alberta Stroke Programme Early CT Score. Lancet 355:1670-1674, 2000.

26. Hill MD, Rowley HA, Adler F, Eliasziw M, Furlan A, Higashida $\mathrm{RT}$, et al. Selection of acute ischemic stroke patients for intraarterial thrombolysis with pro-urokinase by using ASPECTS. Stroke 34:1925-1931, 2003.

27. Lev M, Farkas J, Gemmete J, Hossain S, Hunter G, Koroshetz W, et al. Acute stroke: improved nonenhanced CT detection-benefits of soft-copy interpretation by using variable window width and center level settings. Radiology 213:150-155, 1999.

28. Hunter GJ, Hamberg LM, Ponzo JA, Huang-Hellinger FR, Morris PP, Rabinov J, et al. Assessment of cerebral perfusion and arterial anatomy in hyperacute stroke with three-dimensional functional CT: early clinical results. AJNR Am J Neuroradiol 19:29-37, 1998.

29. Eastwood JD, Lev MH, Provenzale JM. Perfusion CT with iodinated contrast material. AJR Am J Roentgenol 180:3-12, 2003.

30. Lev MH, Segal AZ, Farkas J, Hossain ST, Putman C, Hunter GJ, et al. Utility of perfusion-weighted CT imaging in acute middle cerebral artery stroke treated with intra-arterial thrombolysis: prediction of final infarct volume and clinical outcome. Stroke 32: 2021-2028, 2001.

31. Wintermark M, Reichhart M, Thiran JP, Maeder P, Chalaron M, Schnyder P, et al. Prognostic accuracy of cerebral blood flow measurement by perfusion computed tomography, at the time of emergency room admission, in acute stroke patients. Ann Neurol 51:417-432, 2002.

32. Schramm P, Schellinger PD, Klotz E, Kallenberg K, Fiebach JB, Kulkens S, et al. Comparison of perfusion computed tomography and computed tomography angiography source images with perfusion-weighted imaging and diffusion-weighted imaging in patients with acute stroke of less than 6 hours' duration. Stroke 35:1652-1658, 2004.

33. Grond M, Rudolf J, Schneweis S, Terstegge K, Sobesky J, Kracht $\mathrm{L}$, et al. Feasibility of source images of computed tomographic angiography to detect the extent of ischemia in hyperacute stroke. Cerebrovasc Dis 13:251-256, 2002.

34. Nabavi DG, Cenic A, Dool J, Smith RM, Espinosa F, Craen RA, et al. Quantitative assessment of cerebral hemodynamics using CT: stability, accuracy, and precision studies in dogs. J Comput Assist Tomogr 23:506-515, 1999.

35. Roberts HC, Roberts TP, Smith WS, Lee TJ, Fischbein NJ, Dillon WP. Multisection dynamic CT perfusion for acute cerebral isch- emia: the "toggling-table" technique. AJNR Am J Neuroradiol 22:1077-1080, 2001.

36. Wintermark M, Smith WS, Ko NU, Quist M, Schnyder P, Dillon WP. Dynamic perfusion CT: optimizing the temporal resolution and contrast volume for calculation of perfusion CT parameters in stroke patients. AJNR Am J Neuroradiol 25:720-729, 2004.

37. Wintermark M, Thiran JP, Maeder P, Schnyder P, Meuli R. Simultaneous measurement of regional cerebral blood flow by perfusion CT and stable xenon CT: a validation study. AJNR Am J Neuroradiol 22:905-914, 2001.

38. Koenig M, Klotz E, Luka B, Venderink DJ, Spittler JF, Heuser L. Perfusion CT of the brain: diagnostic approach for early detection of ischemic stroke. Radiology 209:85-93, 1998.

39. Reichenbach JR, Rother J, Jonetz-Mentzel L, Herzau M, Fiala A, Weiller C, et al. Acute stroke evaluated by time-to-peak mapping during initial and early follow-up perfusion CT studies. AJNR Am J Neuroradiol 20:1842-1850, 1999.

40. Esteban JM, Cervera V. Perfusion CT and angio CT in the assessment of acute stroke. Neuroradiology 46:705-715, 2004.

41. Eastwood JD, Lev MH, Wintermark M, Fitzek C, Barboriak DP, Delong DM, et al. Correlation of early dynamic CT perfusion imaging with whole-brain MR diffusion and perfusion imaging in acute hemispheric stroke. AJNR Am J Neuroradiol 24:1869-1875, 2003.

42. Bammer R, Skare S, Newbould R, Liu C, Thijs V, Ropele S, Clayton DB, Krueger G, Moseley ME, Glover GH. Foundations of advanced magnetic resonance imaging. NeuroRx 2:167-196, 2005.

43. Lev MH, Farkas J, Rodriguez VR, Schwamm LH, Hunter GJ, Putman CM, et al. CT angiography in the rapid triage of patients with hyperacute stroke to intraarterial thrombolysis: accuracy in the detection of large vessel thrombus. J Comput Assist Tomogr 25:520-528, 2001.

44. Knauth M, von Kummer R, Jansen O, Hahnel S, Dorfler A, Sartor $\mathrm{K}$. Potential of CT angiography in acute ischemic stroke. AJNR Am J Neuroradiol 18:1001-1010, 1997.

45. Koelemay MJ, Nederkoorn PJ, Reitsma JB, Majoie CB. Systematic review of computed tomographic angiography for assessment of carotid artery disease. Stroke 35:2306-2312, 2004.

46. Anderson GB, Ashforth R, Steinke DE, Ferdinandy R, Findlay JM $\mathrm{CT}$ angiography for the detection and characterization of carotid artery bifurcation disease. Stroke 31:2168-2174, 2000.

47. Henson JW Nogueira RG, Covarrubias DJ, Gonzalez RG, Lev MH. Gadolinium contrast CT angiography of the circle of willis and neck. AJNR Am J Neuroradiol 25:969-972, 2004.

48. Graf J, Skutta B, Kuhn FP, Ferbert A. Computed tomographic angiography findings in 103 patients following vascular events in the posterior circulation: potential and clinical relevance. J Neurol 247:760-766, 2000.

49. Firlik AD, Kaufmann AM, Wechsler LR, Firlik KS, Fukui MB, Yonas H. Quantitative cerebral blood flow determinations in acute ischemic stroke. Relationship to computed tomography and angiography. Stroke 28:2208-2213, 1997

50. Mullins ME, Schaefer PW, Sorensen AG, Halpern EF, Ay H, He $\mathrm{J}$, et al. CT and conventional and diffusion-weighted MR imaging in acute stroke: study in 691 patients at presentation to the emergency department. Radiology 224:353-360, 2002.

51. Gonzalez RG, Schaefer PW, Buonanno FS, Schwamm LH, Budzik RF, Rordorf G, et al. Diffusion-weighted MR imaging: diagnostic accuracy in patients imaged within 6 hours of stroke symptom onset. Radiology 210:155-162, 1999.

52. Ay H, Buonanno FS, Rordorf G, Schaefer PW, Schwamm LH, Wu $\mathrm{O}$, et al. Normal diffusion-weighted MRI during stroke-like deficits. Neurology 52:1784-1792, 1999.

53. Ay H, Oliveira-Filho J, Buonanno FS, Ezzeddine M, Schaefer PW, Rordorf G, et al. Diffusion-weighted imaging identifies a subset of lacunar infarction associated with embolic source. Stroke 30:2644-2650, 1999.

54. Gerraty RP, Parsons MW, Barber PA, Darby DG, Desmond PM, Tress BM, et al. Examining the lacunar hypothesis with diffusion and perfusion magnetic resonance imaging. Stroke 33:2019-2024, 2002. 
55. Roh JK, Kang DW, Lee SH, Yoon BW, Chang KH. Significance of acute multiple brain infarction on diffusion-weighted imaging. Stroke 31:688-694, 2000.

56. Kang DW, Chalela JA, Ezzeddine MA, Warach S. Association of ischemic lesion patterns on early diffusion-weighted imaging with TOAST stroke subtypes. Arch Neurol 60:1730-1734, 2003.

57. Lin W, Lee JM, Lee YZ, Vo KD, Pilgram T, Hsu CY. Temporal relationship between apparent diffusion coefficient and absolute measurements of cerebral blood flow in acute stroke patients. Stroke 34:64-70, 2003.

58. Li F, Silva MD, Liu KF, Helmer KG, Omae T, Fenstermacher JD, et al. Secondary decline in apparent diffusion coefficient and neurological outcomes after a short period of focal brain ischemia in rats. Ann Neurol 48:236-244, 2000.

59. Kidwell CS, Saver JL, Mattiello J, Starkman S, Vinuela F, Duckwiler G, et al. Thrombolytic reversal of acute human cerebral ischemic injury shown by diffusion/perfusion magnetic resonance imaging. Ann Neurol 47:462-469, 2000.

60. Schwamm LH, Koroshetz WJ, Sorensen AG, Wang B, Copen WA, Budzik R, et al. Time course of lesion development in patients with acute stroke: serial diffusion- and hemodynamic-weighted magnetic resonance imaging. Stroke 29:2268-2276, 1998.

61. Baird AE, Benfield A, Schlaug G, Siewert B, Lovblad KO, Edelman RR, et al. Enlargement of human cerebral ischemic lesion volumes measured by diffusion-weighted magnetic resonance imaging. Ann Neurol 41:581-589, 1997.

62. Fiehler J, Knudsen K, Kucinski T, Kidwell CS, Alger JR, Thoma1la G, et al. Predictors of apparent diffusion coefficient normalization in stroke patients. Stroke 35:514-519, 2004.

63. Grandin CB. Assessment of brain perfusion with MRI: methodology and application to acute stroke. Neuroradiology 45:755-766, 2003.

64. Yamada K, Wu O, Gonzalez RG, Bakker D, Ostergaard L, Copen WA, et al. Magnetic resonance perfusion-weighted imaging of acute cerebral infarction: effect of the calculation methods and underlying vasculopathy. Stroke 33:87-94, 2002.

65. Schaefer PW, Hunter GJ, He J, Hamberg LM, Sorensen AG, Schwamm LH, et al. Predicting cerebral ischemic infarct volume with diffusion and perfusion MR imaging. AJNR Am J Neuroradiol 23:1785-1794, 2002.

66. Albers GW. Expanding the window for thrombolytic therapy in acute stroke. The potential role of acute MRI for patient selection. Stroke 30:2230-2237, 1999.

67. Rordorf G, Koroshetz WJ, Copen WA, Cramer SC, Schaefer PW, Budzik RF Jr, et al. Regional ischemia and ischemic injury in patients with acute middle cerebral artery stroke as defined by early diffusion-weighted and perfusion-weighted MRI. Stroke 29:939943, 1998.

68. Karonen JO, Liu Y, Vanninen RL, Ostergaard L, Kaarina Partanen PL, Vainio PA, et al. Combined perfusion- and diffusion-weighted MR imaging in acute ischemic stroke during the 1st week: a longitudinal study. Radiology 217:886-894, 2000.

69. Rother J, Schellinger PD, Gass A, Siebler M, Villringer A, Fiebach $\mathrm{JB}$, et al. Effect of intravenous thrombolysis on MRI parameters and functional outcome in acute stroke $<6$ hours. Stroke 33:2438 2445,2002

69a.Hacke W, Albers G, Al-Rawi Y, Bogousslavsky J, Davalos A, Eliasziw M, et al. The Desmoteplase in Acute Ischemic Stroke Trial (DIAS); a phase II MRI-based 9-hour window acute stroke thrombolysis trial with intravenous desmoteplase. Stroke 36:6673, 2005.

70. Doege CA, Kerskens CM, Romero BI, Brunecker P, Junge-Hulsing $\mathbf{J}$, von Pannwitz W, et al. Assessment of diffusion and perfusion deficits in patients with small subcortical ischemia. AJNR Am J Neuroradiol 24:1355-1363, 2003.

71. Jovin TG, Yonas H, Gebel JM, Kanal E, Chang YF, Grahovac SZ, et al. The cortical ischemic core and not the consistently present penumbra is a determinant of clinical outcome in acute middle cerebral artery occlusion. Stroke 34:2426-2433, 2003.

72. Sorensen A, Copen W, Ostergaard L, Buonnano F, Gonzalez R, Rordof G, et al. Hyperacute stroke: simultaneous measurement of relative cerebral blood volume, relative cerebral blood flow and mean tissue transit time. Radiology 210:519-527, 1999.
73. Schaefer PW, Ozsunar Y, He J, Hamberg LM, Hunter GJ, Sorensen AG, et al. Assessing tissue viability with MR diffusion and perfusion imaging. AJNR Am J Neuroradiol 24:436-443, 2003.

74. Rohl L, Ostergaard L, Simonsen CZ, Vestergaard-Poulsen P, Andersen G, Sakoh M, et al. Viability thresholds of ischemic penumbra of hyperacute stroke defined by perfusion-weighted MRI and apparent diffusion coefficient. Stroke 32:1140-1146, 2001.

75. Grandin CB, Duprez TP, Smith AM, Mataigne F, Peeters A, Oppenheim $\mathrm{C}$, et al. Usefulness of magnetic resonance-derived quantitative measurements of cerebral blood flow and volume in prediction of infarct growth in hyperacute stroke. Stroke 32:11471153, 2001.

76. Butcher K, Parsons M, Baird T, Barber A, Donnan G, Desmond P, et al. Perfusion thresholds in acute stroke thrombolysis. Stroke 34:2159-2164, 2003.

77. Parsons MW, Barber PA, Chalk J, Darby DG, Rose S, Desmond $\mathrm{PM}$, et al. Diffusion- and perfusion-weighted MRI response to thrombolysis in stroke. Ann Neurol 51:28-37, 2002.

78. Wittsack HJ, Ritzl A, Fink GR, Wenserski F, Siebler M, Seitz RJ, et al. MR imaging in acute stroke: diffusion-weighted and perfusion imaging parameters for predicting infarct size. Radiology 222:397-403, 2002.

79. Chalela JA, Kang DW, Luby M, Ezzeddine M, Latour LL, Todd $\mathrm{JW}$, et al. Early magnetic resonance imaging findings in patients receiving tissue plasminogen activator predict outcome: insights into the pathophysiology of acute stroke in the thrombolysis era. Ann Neurol 55:105-112, 2004.

80. Hillis AE, Wityk RJ, Beauchamp NJ, Ulatowski JA, Jacobs MA, Barker PB. Perfusion-weighted MRI as a marker of response to treatment in acute and subacute stroke. Neuroradiology 46:31-39, 2004.

81. Fiebach JB, Schellinger PD, Gass A, Kucinski T, Siebler M, Villringer A, et al. Stroke magnetic resonance imaging is accurate in hyperacute intracerebral hemorrhage: a multicenter study on the validity of stroke imaging. Stroke 35:502-506, 2004.

82. Hermier M, Nighoghossian N. Contribution of susceptibilityweighted imaging to acute stroke assessment. Stroke 35:19891994, 2004.

83. Lee SH, Bae HJ, Kwon SJ, Kim H, Kim YH, Yoon BW, et al. Cerebral microbleeds are regionally associated with intracerebral hemorrhage. Neurology 62:72-76, 2004.

84. Kidwell CS, Saver JL, Carneado J, Sayre J, Starkman S, Duckwiler $\mathrm{G}$, et al. Predictors of hemorrhagic transformation in patients receiving intra-arterial thrombolysis. Stroke 33:717-724, 2002.

85. Nighoghossian N, Hermier M, Adeleine P, Blanc-Lasserre K, Derex L, Honnorat J, et al. Old microbleeds are a potential risk factor for cerebral bleeding after ischemic stroke: a gradient-echo T2weighted brain MRI study. Stroke 33:735-742, 2002.

86. Greer DM, Koroshetz WJ, Cullen S, Gonzalez RG, Lev MH. Magnetic resonance imaging improves detection of intracerebral hemorrhage over computed tomography after intra-arterial thrombolysis. Stroke 35:491-495, 2004.

87. Nederkoorn PJ, van der Graaf Y, Hunink MG. Duplex ultrasound and magnetic resonance angiography compared with digital subtraction angiography in carotid artery stenosis: a systematic review. Stroke 34:1324-1332, 2003.

88. Lev MH, Romero JM, Gonzalez RG. Flow voids in time-of-flight MR angiography of carotid artery stenosis? It depends on the TE! AJNR Am J Neuroradiol 24:2120, 2003.

89. Nederkoorn PJ, Elgersma OE, van der Graaf Y, Eikelboom BC, Kappelle LJ, Mali WP. Carotid artery stenosis: accuracy of contrast-enhanced MR angiography for diagnosis. Radiology 228:677-682, 2003.

90. Pedraza S, Silva Y, Mendez J, Inaraja L, Vera J, Serena J, et al. Comparison of preperfusion and postperfusion magnetic resonance angiography in acute stroke. Stroke 35:2105-2110, 2004.

91. Lin A, Ross BD, Harris K, Wong W. Efficacy of proton magnetic resonance spectroscopy in neurological diagnosis and neurotherapeutic decision making. NeuroRx 2:197-214, 2005. 
92. Nicoli F, Lefur Y, Denis B, Ranjeva JP, Confort-Gouny S, Cozzone PJ. Metabolic counterpart of decreased apparent diffusioncoefficient during hyperacute ischemic stroke: a brain proton magnetic resonance spectroscopic imaging study. Stroke 34:e82-e87, 2003.

93. Fiehler J. Editorial comment-ADC and metabolites in stroke: even more confusion about diffusion? Stroke 34:e87-e88, 2003.

94. Parsons MW, Li T, Barber PA, Yang Q, Darby DG, Desmond PM, et al. Combined (1)H MR spectroscopy and diffusion-weighted MRI improves the prediction of stroke outcome. Neurology 55: 498-505, 2000.
95. Harris AD, Pereira RS, Mitchell JR, Hill MD, Sevick RJ, Frayne R. A comparison of images generated from diffusion-weighted and diffusion-tensor imaging data in hyper-acute stroke. J Magn Reson Imaging 20:193-200, 2004.

96. Lie C, Hirsch JG, Rossmanith C, Hennerici MG, Gass A. Clinicotopographical correlation of corticospinal tract stroke: a colorcoded diffusion tensor imaging study. Stroke 35:86-92, 2004.

97. Kunimatsu A, Aoki S, Masutani Y, Abe O, Mori H, Ohtomo K. Three-dimensional white matter tractography by diffusion tensor imaging in ischaemic stroke involving the corticospinal tract. Neuroradiology 45:532-535, 2003. 